\title{
PROCESS AND SYSTEMS Patient Journey Champions: A project to facilitate delivery of effective board rounds for inpatients, aiding flow through the hospital
}

\author{
Authors: Aaisha Saqib, ${ }^{A}$ William Waugh, ${ }^{B}$ Rebecca Jayasinghe ${ }^{C}$ and Sandip Banerjee ${ }^{D}$
}

Our quality improvement project was designed to enable delivery of high-quality board rounds across the hospital with a view to improving patient flow. We designed a Patient Journey Champion campaign to enable this. As part of our campaign, we ran sessions with junior doctors. These comprised education on the structure of a board round, giving them an insight into the bigger picture on patient flow and eventually aiming to empower them to lead an effective board round on their respective wards, where they would act as 'Patient Journey Champions'. Following the workshops, we audited the quality of board rounds and compared it with baseline. The quality was measured against the hospital standard operating procedure. We noticed an improvement in the quality of board rounds and also a positive effect on length of stay on the acute admissions ward. We anticipate a continuing trend of improvement as this intervention is rolled out across more doctors and other staff groups, although this relies on the need to involve doctors in board rounds and empowering them with the appropriate skills. Simultaneously, work is now being undertaken by matrons of respective wards to upskill ward nurses and other allied healthcare professionals to aid delivery of improved board rounds.

KEYWORDS: Board rounds, patient flow, educational, patient journey champions

DOI: $10.7861 /$ fhj.2020-0021

\section{Introduction}

The challenges around improving patient flow are well known across the NHS, and impact on both staff and service delivery. The pressures of meeting targets and providing good care for patients while maintaining clinical standards often result in departments operating in 'crisis mode'.

Authors: ${ }^{\text {ARoyal }}$ College of Physicians chief registrar, Medway Maritime Hospital, Gillingham, UK; ${ }^{\text {B }}$ foundation year-2 doctor, Medway Maritime Hospital, Gillingham, UK; ${ }^{\text {C }}$ specialist medicine registrar, Medway Maritime Hospital, Gillingham, UK; ${ }^{\text {D }}$ consultant physician and clinical director for specialist medicine, Medway Maritime Hospital, Gillingham, UK
Issues with patient flow can lead to extended emergency department waiting times and extended hospital stay. In our hospital, the majority of beds are occupied by patients deemed 'medically fit', with the total number varying at different time points throughout the year. With increasingly limited resources leading to increasing demands to do more with less, inefficiencies in patient flow must be identified, addressed and eradicated. One such inefficiency in the system could be a low-quality board round.

Board rounds are a summary discussion of the patient journey. They facilitate allocation of the daily tasks required for the journey to progress, and identify and resolve any delays in the patient's hospital stay. This enhances patient experience and reduces the risk factors associated with a prolonged hospital stay. ${ }^{2}$

\section{Methods}

This project utilises a continuous quality improvement (QI) methodology. Twenty wards in the hospital were included, comprising a mixture of medical and surgical wards. Board rounds were graded against a hospital standard operating procedure (supplementary material S1) on a scale from 1-4 (1 = requires significant improvement up to $4=$ very good). Data were collected over several weeks to provide a baseline.

An intervention was implemented in the form of the Patient Journey Champions initiative. These workshops intended to educate junior doctors on the structure of a board round, giving them an insight into the bigger picture on patient flow and eventually aiming to empower them to lead an effective board round on their respective wards, where they would act as 'Patient Journey Champions'. These sessions comprised a small lecture followed by a feedback session with junior doctors, when we listened to their feedback and ideas. One of the aims of the sessions was to encourage engagement from medical teams while empowering them to see the hospital bigger picture and explaining how a high-quality board round can impact on the patient journey in a positive way. Board rounds were continually observed as the intervention was administered to additional groups of doctors.

Simultaneously, work was being undertaken by matrons of respective wards to upskill ward nurses and other allied healthcare professionals to aid delivery of improved board rounds, with the objective of achieving better patient flow within and out of the hospital. 


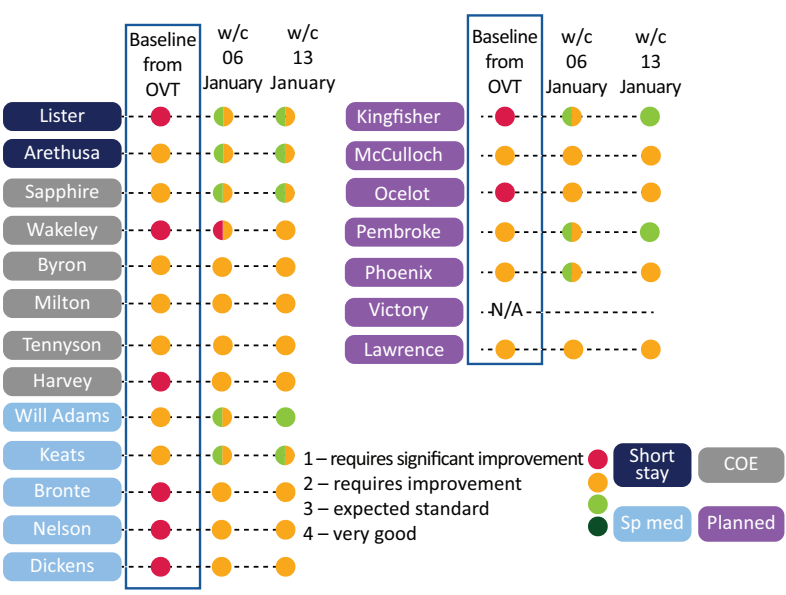

Fig 1. Summary of board round progress across all wards. Note: Lister, Dickens and Ocelot wards did not have board rounds at baseline. $\mathrm{COE}=$ care of the elderly; N/A = not applicable; OVT = one version of truth (baseline data collected over several weeks); Sp med = specialty medicine; $\mathrm{w} / \mathrm{c}=$ week commencing.

\section{Results}

At baseline, all wards were graded at 1 or 2 , with three wards not having board rounds at all. Since interventions have begun, improvement has been seen in 14 of 20 wards, and the most recent data show 12 wards at grade 2 , four wards at grade 2.5 and three wards at grade 3 , suggesting that our intervention led to an improvement in board rounds soon after the teaching sessions were delivered (Fig 1).

Before the start of formal board round training workshops with staff, we did not have board rounds on our acute wards. On some specialty medicine wards, there were no decisions being made on expected dates of discharge due to a high percentage of patients being medically fit. We also observed in our baseline data collection that there was a lack of engagement from the medical teams on some wards. Following the intervention, the greatest improvement was seen in engagement from medical teams, the board round leadership improved and actions from the discussions started to be appropriately allocated. Following the intervention, we saw a significant reduction in length of stay in our acute medical wards, which did not have a structured board round set up at the start of this project (Fig 2). The data on length of stay from one of the acute wards suggests an increased length of stay during winter months. We found that this was due to issues with lack of movement from acute medical wards to specialist or frailty beds. This gets worse in winter months as the hospital struggles to discharge medically fit patients to the community, leading to an increase in lengths of stay.

\section{Discussion and future recommendations}

While conducting sessions with doctors, we also collated feedback. One idea put forward was to move the board rounds to the latter half of the morning in order to get clear medical plans following the ward round. This will be discussed with the executive team, matrons and medical director. Also, as the work on improving board rounds was being undertaken, we realised that the commitment to implementation will take several weeks given the nature of shift working, hence it will be an ongoing project.

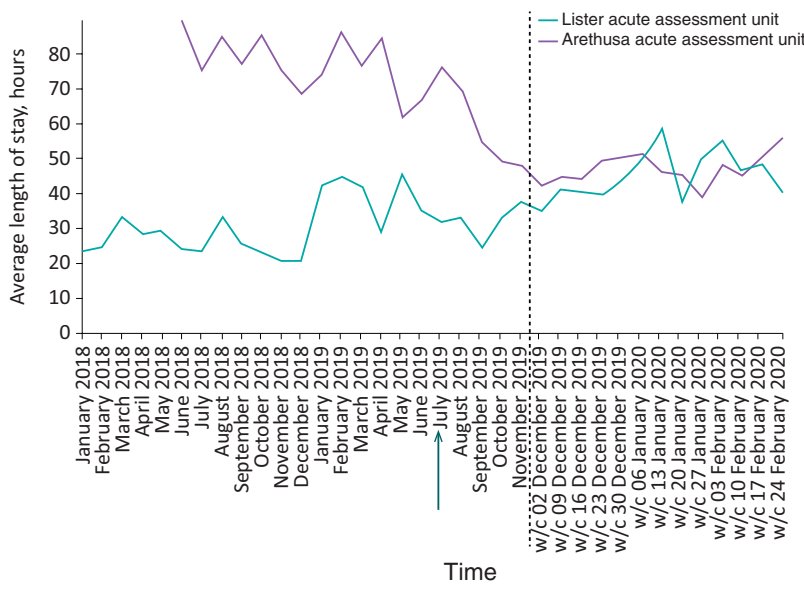

Fig 2. Average length of stay on acute assessment unit wards by month then week, 01 January 2018 - 01 March 2020. The dark teal arrow indicates the time of the intervention. $w / c=$ week commencing.

In teaching sessions, it is not always possible to capture the full cohort of doctors and as they work on a rotational basis, there is an ongoing need to role model good practice when handing over care at board rounds.

We anticipate a continuing trend of improvement as this intervention is rolled out across more doctors and other staff groups, although this relies on the need to involve more doctors in board rounds and empowering them with the appropriate skills.

Continuity of junior doctors and senior nurses also impacts on implementation and motivation for the changes. Regular team meetings to discuss progress will help address this. We also learned from delivering these sessions that providing shared ownership of the process will promote further sustainability. Future cycles will focus on further refining the process, including timing of electronic discharge notices and readdressing issues around the daily timing of board round discussions, to facilitate greater team working at ward level.

\section{Supplementary material}

Additional supplementary material may be found in the online version of this article at www.rcpjournals.org/fhj:

S1 - Standard operating procedure for board rounds, Medway NHS Foundation Trust.

\section{References}

1 NHS England. Transforming urgent and emergency care services in England. NHS, 2015. www.england.nhs.uk/wp-content/uploads/ 2015/06/trans-uec.pdf [Accessed 31 July 2020].

2 NHS Improvement. Internal professional standards (IPS): Board rounds. NHS, 2018. https://improvement.nhs.uk/documents/1696/Board_ Round_Internal_Professional_Standards_FINAL_webversion02.01.18_ ed_15.01.18.pdf [Accessed 31 July 2020].

Address for correspondence: Dr Aaisha Saqib, Medway Maritime Hospital, Windmill Road, Gillingham, Kent ME7 5NY, UK.

Email: aaishasaqib@nhs.net 